\title{
High-temperature ferromagnetism in dilute magnetic oxides
}

\author{
J. M. D. Coey ${ }^{\mathrm{a})}$ \\ Physics Department, Trinity College, Dublin 2, Ireland
}

(Received 27 September 2004; accepted 7 November 2004; published online 6 May 2005)

\begin{abstract}
High temperature ferromagnetism in thin films of dilute magnetic oxides is a widespread phenomenon, of which there appear to be two sources. One is the contribution of the $3 d$ dopant ions themselves, the other is related to crystal defects in the interface region. The latter contributes a magnetic moment of $100-400 \mu_{\mathrm{B}}$ per square nanometer of substrate area, which is largely independent of film thickness or dopant concentration. It is suggested that the defects are two-electron or two-hole centers which have a spin triplet as ground state or low-lying excited state. $\mathrm{HfO}_{2}$, an example of the former, is ferromagnetic even when undoped. In $\mathrm{ZnO}$ or $\mathrm{SnO}_{2}$, examples of the latter, the magnetic dopant may stabilize the spin triplet by exchange. (C) 2005 American Institute of Physics. [DOI: 10.1063/1.1849054]
\end{abstract}

Following the discovery by Matsumoto et al. in 2001 of high-temperature ferromagnetism in an anatase film doped with $2 \%$ of cobalt, ${ }^{1}$ it is gradually becoming clear that ferromagnetism in lightly doped oxides and nitrides is a widespread and hitherto-unsuspected phenomenon. ${ }^{2,3}$ The main characteristics of these materials may be summarized as follows:

- The ferromagnetism is usually only found in thin films, at doping levels below the percolation threshold for nearest-neighbor cation interactions.

- The films may be insulating or semiconductiing, but if semiconducting they are often $n$ type.

- The moment per dopant cation decreases as the dopant concentration $x$ increases, but it may exceed the spinonly value at low concentrations.

- Curie temperatures can be well in excess of room temperature. $T_{C}$ does not seem to scale with dopant concentration $x$.

- Unusual anisotropy of the magnetization relative to the film plane is often observed.

The results are not very reproducible, with different preparation methods leading to different moments or ordering temperatures. In some films, notably of $\mathrm{TiO}_{2}$, secondary parasitic phases have definitely been shown to be present and they may contribute to the magnetization, especially in systems such as Co-doped $\mathrm{TiO}_{2}$, which exhibit a low solid solubility of the dopant cation. However, this cannot be a general explanation of the ferromagnetism for two reasons:

1. The moment per cation at low concentrations can exceed that of any known ferromagnetic phase. ${ }^{4-9}$

2. The anisotropy of the saturation magnetization is unlike that of any known ferromagnetic phase., ${ }^{5,6,9-11}$

It seems that we are looking at a generic phenomenon, which challenges our current understanding of magnetic order in solids. This is epitomized in the " $\boldsymbol{m}$ - $J$ paradigm," where a magnetic moment $\boldsymbol{m}$ is associated with the atoms or ions in a metal or insulator, and an exchange interaction $J$ couples the atomic spins parallel or antiparallel. In the high-temperature oxide or nitride ferromagnets, it is natural to assume that the

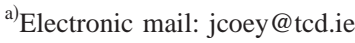

moments are associated with the spins of dopant ions, and therefore that there must be some unusually strong exchange interaction, which couples the spins parallel. In theories of ferromagnetism in dilute systems, the Curie temperature usually varies as $x$ or as $\sqrt{ }$, where $x$ is the dopant concentration. $^{12,13}$ A Curie temperature well in excess of room temperature in an oxide with $x=5 \%$ therefore implies exchange interactions that are stronger than those in any known ferromagnet. Here we present evidence that suggests the $\boldsymbol{m}$ assumption, at least, is mistaken.

Table I summarizes some reports of giant moments in oxides, following the original report of $7.5 \mu_{\mathrm{B}}$ per ion for Co-doped $\mathrm{SnO}_{2}$ by Ogale et al. ${ }^{4}$ It is rare to encounter the ionic spin-only moment in any ferromagnetic $3 d$ solid. Metals and ferromagnetic insulators usually have net moments per ion that are less than about half the spin-only values. It is only in a few half-metallic $3 d$ systems that the full spin-only moment is encountered, for example in the ferromagnetic mixed-valence manganites such as $\left(\mathrm{La}_{0.7} \mathrm{Sr}_{0.3}\right) \mathrm{MnO}_{3}$. Rare earth metals and alloys have atomic-like localized $4 f$ cores. Giant moments in metals are known for some dilute alloys where dopant atoms are inserted into a host with an enhanced Pauli susceptibility, CoPd, for instance. But in the present case, the host oxide is diamagnetic in pure bulk form. Orbital moments are largely quenched for $3 d$ impurities in oxides. There must be another source of the magnetism.

In Fig. 1, we plot the moments measured as a function of the product $x t$ for a large number of ferromagnetic films prepared by pulsed-laser deposition in our laboratory; $t$ is the film thickness. The data are for different matrices, different dopings, and different substrates. Moments are all expressed

TABLE I. Giant magnetic moments in oxide thin films.

\begin{tabular}{lllrl}
\hline \hline Oxide & Dopant & $x$ & $m\left(\mu_{\mathrm{B}} /\right.$ dopant $)$ & Reference \\
\hline $\mathrm{SnO}_{2}$ & Co & 0.05 & 7.5 & 4 \\
& $\mathrm{Co}$ & 0.01 & 5.0 & 5 \\
& $\mathrm{Mn}$ & 0.003 & 20 & 5 \\
$\mathrm{ZnO}$ & $\mathrm{Co}$ & 0.02 & 5.9 & 6 \\
$\mathrm{TiO}_{2}$ & $\mathrm{~V}$ & 0.05 & 4.2 & 7 \\
$\mathrm{HfO}_{2}$ & Co & 0.05 & 6.8 & 8 \\
& Co & 0.02 & 30 & 9 \\
\hline \hline
\end{tabular}




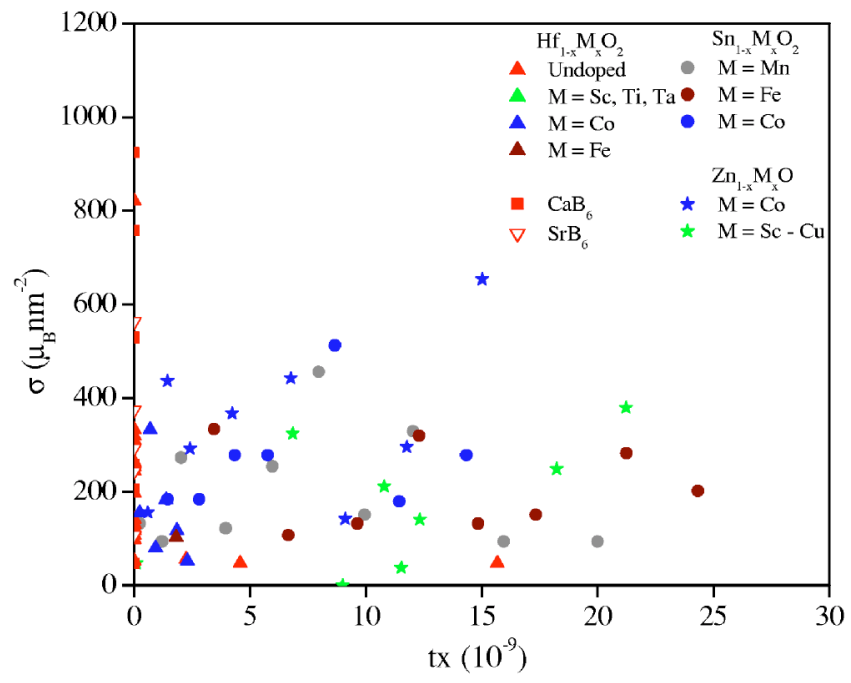

FIG. 1. Room-temperature magnetic moments in doped and undoped oxide thin films as a function of the product of doping $x$ and film thickness $t$.

per unit substrate area using the handy units of Bohr magnetons per square nanometer, $\mu_{\mathrm{B}} \mathrm{nm}^{-2}$. Despite the scatter, neither the overall plot, nor any restricted data sets where the matrix, dopant, or substrate is kept constant show any sign of a proportionality with $x$ or $t$. The films need only be about 10 $\mathrm{nm}$ thick to exhibit the full moment. The statement that can be made from these data is that the room-temperature moment per unit substrate area in $90 \%$ of our films falls in the range $100-400 \mu_{\mathrm{B}} \mathrm{nm}^{-2}$. It is also noteworthy that a moment of this magnitude is found in some films that contain no $3 d$ dopant ions- $\mathrm{HfO}_{2}, \mathrm{ZrO}_{2}, \mathrm{CaB}_{6}, \mathrm{SrB}_{6}$, and Sc-doped $\mathrm{ZnO}-$ but not in others- $\mathrm{CaO}, \mathrm{MgO}, \mathrm{ZnO}, \mathrm{SnO}_{2}, \mathrm{TiO}_{2}$, and $\mathrm{Sn}$-doped $\mathrm{In}_{2} \mathrm{O}_{3}$ - which are nonmagnetic, as one would normally expect.

An example of the data reduction for a typical $\mathrm{HfO}_{2}$ film is shown in Fig. 2. There is a diamagnetic signal from the substrate, which is three orders of magnitude more massive than the film deposited on it. The film was deposited on sapphire, which contains 2 ppm Cr, 3 ppm Mn but no measurable $\mathrm{Fe}$ or $\mathrm{Co}(<1 \mathrm{ppm})$ from a nominally $5 \mathrm{~N}$ pure $\mathrm{HfO}_{2}$ target which actually contained 63 ppm of iron. The substrate

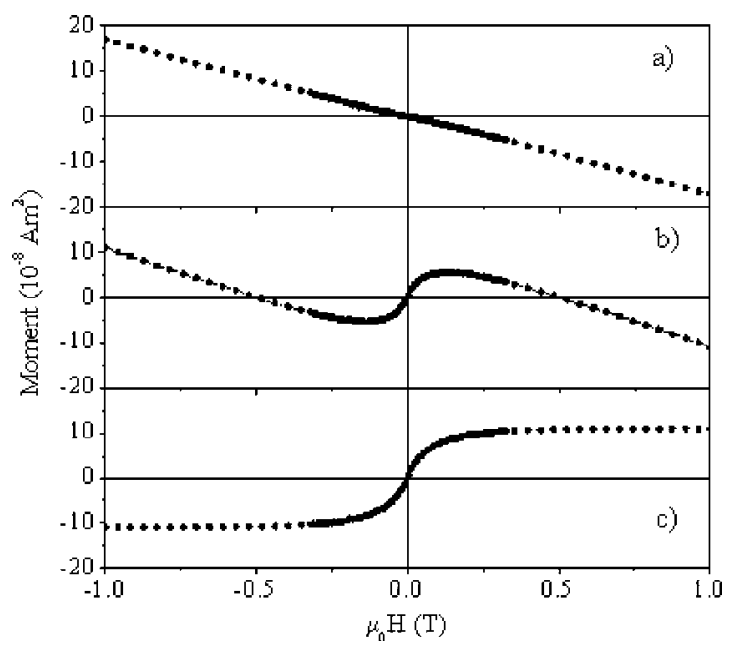

FIG. 2. Typical data reduction for a ferromagnetic oxide film on a diamagnetic substrate.

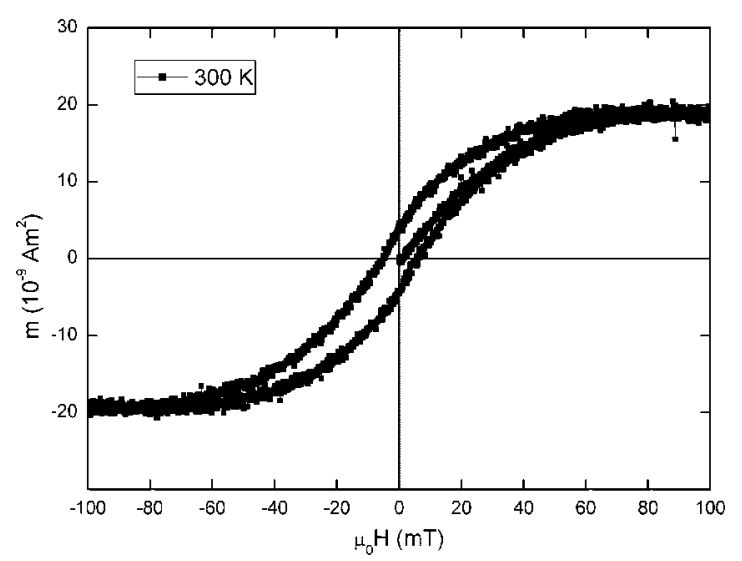

FIG. 3. Hysteresis for a $\mathrm{HfO}_{2}$ film measured at room temperature.

signal is subtracted off using the slope between 3 and $5 \mathrm{~T}$. This procedure gives the extrapolated spontaneous magnetization, but not the high-field slope. The moment of the film in Fig. 2 is equivalent to $0.12 \mu_{\mathrm{B}}$ per hafnium, which would correspond to more than 2 at. \% of high-spin ferromagnetic impurity in the film ( $>5 \%$ of metallic iron or cobalt). We find no evidence whatsoever of this in any energy dispersive $\mathrm{x}$-ray analysis (EDAX) or inductively coupled plasma (ICP) analysis, or on scanning the interface region with a subnanometer analytical probe in (TEM). The ferromagnetism is weakly hysteretic, as shown in Fig. 3. Coercivity is $2-5 \mathrm{mT}$, and the remanance:saturation ratio is $3 \%-9 \%$. There is no sign of pinning on the initial magnetization curve. The $4.2 \mathrm{~K}$ loop is similar.

What then is the source of magnetism in these films? It is not simply the dopant cations, although they may contribute. There is another source, which is "switched on" by the presence of magnetic cations in nonmagnetic matrices such as $\mathrm{ZnO}$ or $\mathrm{SnO}_{2}$, and spontaneously present independent of doping for $\mathrm{HfO}_{2}$ or $\mathrm{CaB}_{6}$.

The lack of thickness dependence provides a clue. It is only in the thinnest films $(<10 \mathrm{~nm})$ that the moment begins to disappear. In cases where the saturation magnetization is independent of field direction applied parallel or perpendicular to the film, an anisotropy field of 0.2-0.6 $\mathrm{T}$ has been deduced from the initial slopes of the magnetization curves. ${ }^{14}$ Attributing this to shape anisotropy, the polarization of $0.2-0.6 \mathrm{~T}$, together with the moment of $\approx 200 \mu_{\mathrm{B}} \mathrm{nm}^{-2}$ indicates that the magnetic layer is about 10 $\mathrm{nm}$ thick. In the hexaborides at least, the moment depends on the substrate-compound combination so the ferromagnetic layer is probably at the substrate/film interface, rather than at the free surface of the film. ${ }^{14}$ There are inevitably defects at the interface, so it is to the defects we look for the source of the magnetism.

Figure 4 shows a lattice image of a typical $\mathrm{HfO}_{2}$ film. There is a nanoscale polycrystalline structure near the interface, with columnar growth. The relevant defects might lie in the numerous grain boundaries, but many such images are needed of films with different magnetic properties. Nanoscale compositional analysis of the interface region in several $\mathrm{HfO}_{2}$ films showed no evidence for the presence of ferromagnetic transition metals $M=\mathrm{Fe}, \mathrm{Co}$, or $\mathrm{Ni}$. The $M / \mathrm{Hf}$ ratio was less than $10^{-2}$. 


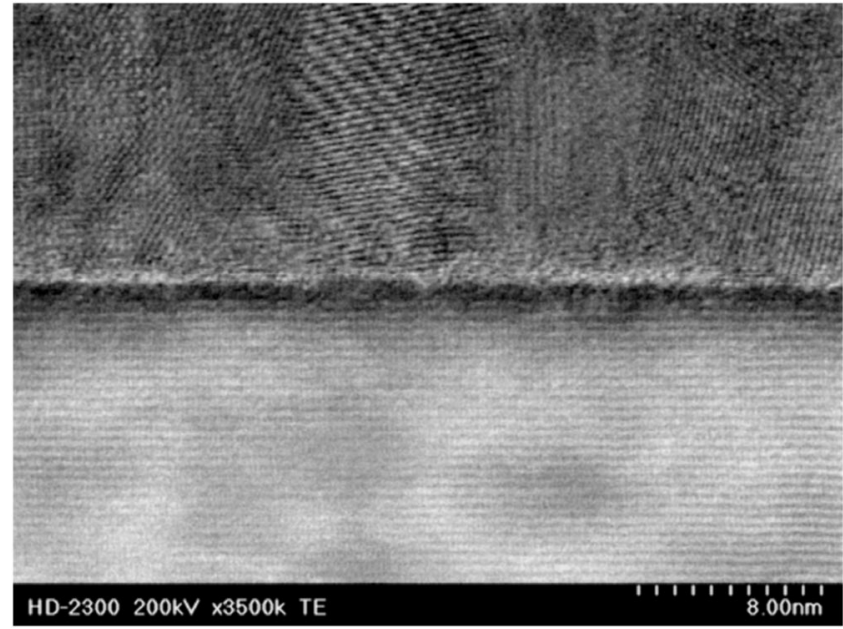

FIG. 4. Bright-field lattice image of $\mathrm{HfO}_{2}$ film on an $\mathrm{Al}_{2} \mathrm{O}_{3}$ substrate, obtained using an Hitachi 2300 STEM.

It has long been known that defects in oxides, which trap two electrons or holes, can have a spin triplet as the ground state or as a low-lying excited state. Such centers in monoxides include the $V^{0}$ cation vacancy center with two holes, and the $F_{t}$ center, which is a pair of singly occupied $F$ centers. ${ }^{15}$ The case of a cation vacancy in $\mathrm{CaO}$ was calculated by Elfimof et al. ${ }^{16}$ who found a triplet two-hole molecular ground state, which ordered magnetically.

The interaction with a magnetic dopant cation is shown schematically in Fig. 5. In $\mathrm{ZnO}$ for example, two-electron defects should have a singlet ground state since undoped $\mathrm{ZnO}$ films show no ferromagnetic properties. A dopant cation with a $3 d$ shell that is half full or more will couple with its moment antiparallel to that of the triplet, and may stabilize the triplet defect state. In $\mathrm{HfO}_{2}$ however, the triplet must be the ground state in the undoped film, and the moment should initially be reduced by Co doping (Fig. 1) because the dopant cation has only unoccupied minority-spin $d$ states. The model requires rather high defect concentrations in the interface region. Assuming an oxygen density of $60 \mathrm{~nm}^{-3}$, a magnetic layer thickness of $10 \mathrm{~nm}$ and $2 \mu_{\mathrm{B}}$ per defect, a moment of $200 \mu_{\mathrm{B}} \mathrm{nm}^{-2}$ gives a defect density of $17 \%$, assuming the defects are oxygen vacancies.

Next we need some interaction to couple the moments together. If the magnetization is concentrated in the interface region, as suggested, it is not necessary that the interaction be so strong and long-range as it would have to be if the moment was distributed uniformly throughout the film. The average defect spacing in the example is $0.5 \mathrm{~nm}$. The wave function of the electrons associated with these defects may occupy extended Bohr orbits on account of the relatively high dielectric constant of the matrix. In the dilute limit, they form bound magnetic polarons, and the ferromagnetism can be regarded as a polaron percolation effect. ${ }^{12,13}$ At higher

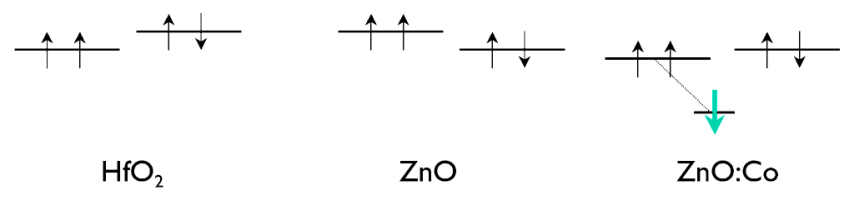

FIG. 5. Singlet and triplet two-electron/hole states in oxides (schematic). concentrations, they overlap to form an impurity band which is quite distinct from that formed by any dopants that may be present. The nature of the magnetic coupling of such defects needs to be investigated, especially in view the huge anisotropy of the saturation magnetization in some cases, ${ }^{5,6,9-11}$ which points to magnetic moments of largely orbital character. It is worth noting that a huge, strongly-anisotropic susceptibility has been observed for polyalanine molecules absorbed on a gold-plated silicon wafer. ${ }^{17}$ Triplet pairing of electrons transferred from the substrate and roomtemperature Bose-Einstein condensation of the spin triplets have been suggested as an explanation. ${ }^{18}$

Dilute magnetic oxides combine can combine optical transparency with a high Curie temperature and semiconducting behaviour. These are very attractive properties for spin electronic applications, but it remains to be established whether or not these controversial materials actually exhibit any of the useful magnetoresistive or magneto-optic properties of their low-Curie-temperature counterparts such as $\left(\mathrm{Ga}_{1-x} \mathrm{Mn}_{x}\right)$ As. It may be necessary to use films about $10 \mathrm{~nm}$ thick in order to observe such effects. In any case, the materials pose a challenge to our understanding of magnetism in solids because of the Curie temperatures far in excess of room temperature in systems containing only a few percent of magnetic ions, or even none at all.

This work was supported by Science Foundation Ireland, as part of the CINSE Project. The author is grateful to many coworkers, including L. S. Dorneles, C. B. Fitzgerald, R. Gunning, J. G. Lunney, M. Moliner, P. Stamenov, M. Venkatesan, and Y. Zhou. M. Konno of Hitachi Science Systems Ltd. kindly took the STEM image.

${ }^{1}$ Y. Matsumoto, M. Murakami, T. Shono, T. Hasegawa et al., Science 292, 854 (2001).

${ }^{2}$ W. Prellier, A. Fouchet, and B. Mercey, J. Phys.: Condens. Matter 15 R1583 (2003).

${ }^{3}$ S. J. Pearton et al., J. Appl. Phys. 93, 1 (2003).

${ }^{4}$ S. B. Ogale, R. J. Choudhary, J. P. Buban, S. E. Lofland et al., Phys. Rev. Lett. 91, 077205 (2003).

${ }^{5}$ C. B. Fitzgerald, M. Venkatesan, and J. M. D. Coey (unpublished).

${ }^{6}$ M. Venkatesan, C. B. Fitzgerald, J. G. Lunney, and J. M. D. Coey, Phys. Rev. Lett. 93, 177206 (2004).

${ }^{7}$ S. Hong et al., Appl. Phys. Lett. 84, 2602 (2004).

${ }^{8}$ M. S. Ramachandra Rao, S. Dhar, S. J. Welz, S. B. Ogale et al., condmat/0405378 (2004).

${ }^{9}$ J. M. D. Coey, M. Venkatesan, P. Stamenov, C. B. Fitzgerald, and L. S. Dorneles, Phys. Rev. B (to be published).

${ }^{10}$ J. M. D. Coey, M. Venkatesan, P. Stamenov, C. B. Fitzgerald, and L. S. Dorneles, J. Magn. Magn. Mater. (to be published).

${ }^{11}$ M. Venkatesan, C. B. Fitzgerald, and J. M. D. Coey, Nature (London) 430, 630 (2004).

${ }^{12}$ J. M. D. Coey, M. Venkatesan, and C. B. Fitzgerald, Nat. Mater. (to be published).

${ }^{13}$ S. Das Sarma, E. W. Hwang, and A. Kaminski, Phys. Rev. B 67, 155201 (2003).

${ }^{14}$ L. S. Dorneles, M. Venkatesan, M. Moliner, J. G. Lunney, and J. M. D. Coey, Appl. Phys. Lett. 85, 6377 (2004).

${ }^{15}$ A. M. Stoneham, Theory of Defects in Solids, Chap. 16 (Clarendon Press, Oxford, 1975)

${ }^{16}$ I. S. Elfimov, S. Yunoki, and G. A. Sawatzky, Phys. Rev. Lett. 89, 216403 (2002).

${ }^{17}$ I. Carmeli, G. Lefitus, R. Naaman, S. Reich, and Z. Vager, J. Chem. Phys. 118, 10372 (2003).

${ }^{18}$ Z. Vager and R. Naaman, Phys. Rev. Lett. 92, 087502 (2004). 